\title{
IDENTIFICATION AND ANALYSIS OF REQUIREMENTS FOR A WEB PLATFORM FOR MANAGING EDUCATIONAL ESCAPE ROOMS
}

\author{
S. López-Pernas, A. Gordillo, E. Barra, J. Quemada \\ Universidad Politécnica de Madrid (SPAIN)
}

\begin{abstract}
Educational escape rooms demand a great effort on the part of instructors, not only when designing and building the activities, but also when the time comes to conduct them. Although previous works have presented tools to ease the creation of educational puzzle-like games, no prior studies have reported on the use, development or design of any tool for managing educational escape rooms.

A tool of this kind would help teachers overcome one of the main barriers they face when trying to incorporate these activities into their teaching. This work identifies and analyses for the first time the requirements that a platform for managing educational escape rooms should satisfy. A platform meeting all the requirements gathered would be able to help teachers in all the steps of the process of conducting an educational escape room, including configuration, integration of the activity into a virtual learning environment, student registration, team creation, control of activity execution (management of resources, narrative events and gamification elements during the activity), progress monitoring, hint delivery, assessment, and gathering of student feedback and data on learning effectiveness.
\end{abstract}

Keywords: Educational escape rooms, gamification, educational software.

\section{INTRODUCTION}

Escape rooms are a new type of leisure activity in which "players discover clues, solve puzzles, and accomplish tasks in one or more rooms in order to accomplish a specific goal (usually escaping from the room) in a limited amount of time" [1]. Due to the ability of these activities to foster teamwork, leadership, creative thinking, and communication in a way that is engaging for students [2]-[7], they have drawn the attention of educators. Moreover, teachers can design escape rooms in such a way that they require students to master the course materials in order to solve their puzzles, creating what is known as educational escape rooms [8]. Despite little research work has been done on educational escape rooms due to their novelty, different researchers have reported on experiences in using this sort of activities in a wide variety of fields including nursing [9], [10], medicine [11], [12], pharmacy [13]-[16], physiotherapy [17], chemistry [18], computer programming [8], computer networks [19], and mathematics [20].

Prior works agree that educational escape rooms demand a great effort on the part of instructors, not only when designing and building the activities, but also when the time comes to conduct them [8], [10], [14]. This fact has also been observed by the authors who, after conducting several educational escape rooms in large programming courses at Universidad Politécnica de Madrid (such as the one reported in [8]), are convinced that this effort is one of the main barriers to the widespread of educational escape rooms. The process of conducting an educational escape room is laborious. First, prior to the activity taking place, students must enrol in the escape room and form teams, and teachers must provide students with a set of instructions that they need to take into account prior to participating. The day of the activity, teachers need to take attendance and provide each team with all the assets needed for the escape room. Additionally, it is common to play an introductory video, audio or other media resource right before the beginning of the activity explaining the task to be accomplished and the narrative of the escape room. Once the escape room activity starts, it is important that everyone is always aware of the time they have left to complete it. During the activity, students can get stuck when solving puzzles and thus they may need to request hints as well as to ask for help. Providing the necessary hints and help in a timely manner during an educational escape room can become cumbersome if the student-teacher ratio is high [8], [13], [14]. Moreover, teachers may need to intervene in different ways during the activity. For instance, they might need to help lagging teams by giving them a free hint, or they might want to play an additional video at a specific moment of the activity as a part of the narrative. After the escape room has been completed by the students, teachers usually need to grade the activity. Given that it may be unfair to grade students based only on whether they attend or successfully complete the escape room, teachers could need 
more accurate grading, which requires to closely monitor the progress of each student. However, such monitoring is very difficult or even infeasible in crowded classrooms. Lastly, gathering students' feedback and testing if they have acquired any knowledge are additional aspects that need to be considered after conducting an educational escape room. Taking into account all the actions that need to be performed when conducting an educational escape room, it becomes clear that a system capable of helping instructors with the non-trivial task of running an educational escape room would be a great contribution to the educational community.

Some attempts have been made to provide tools and other resources to ease the creation of educational puzzle-like activities [21]-[23]. However, no prior studies have reported on the use, development or design of any tool for managing educational escape rooms. Tools like such have been successfully used in the past in order to reduce the burden of orchestrating other types of game-based activities in the classroom such as Trivia [24] and Kahoot [25]. Some of the characteristics that these tools feature include customisation of the activity content, a friendly user interface to control the flow of the activity in class, and easy grading. These aspects are crucial for conducting educational escape rooms as well, in addition to other specific aspects that are unique to this sort of activities.

This work identifies and analyses for the first time the requirements that should be taken into account for developing a platform for managing educational escape rooms. The requirement elicitation described in this work is based on past educational escape room experiences conducted by the authors (such as [8]) and other experiences reported in the literature, which provided a comprehensive view of the distinctive characteristics that educational escape rooms have. A platform meeting all the requirements gathered would be able to help teachers in all the steps of the process of conducting an educational escape room: integration of the activity into a VLE (Virtual Learning Environment), student registration, team creation, control of activity execution (management of resources, narrative events, and gamification elements during the activity), progress monitoring, hint management, assessment of the activity, and gathering of student feedback and data on learning effectiveness. The requirements identified in this work will serve as a starting point for the development of a platform that not only facilitates instructors' work when conducting educational escape rooms but also allows for the creation of better experiences. Thus, this work contributes to the popularisation of these learning activities in educational contexts.

\section{REQUIREMENT ELICITATION}

This section describes all the requirements that a platform for managing educational escape rooms should satisfy that have been identified in this work. The requirements have been divided into four groups, each of them related to one of the phases involved in the management of an educational escape room, taking into account the interactions with the platform from the point of view of both students and teachers. Figure 1 shows a summary of all the requirements.

\subsection{Creation of the educational escape room profile in the platform}

The first step in the management of an educational escape room through the platform should be creating a profile in the platform that includes all the information needed for running it. It should be pointed out that this step is supposed to be carried out by teachers after designing and building the educational escape room itself (i.e. all the puzzles and resources involved in the activity) but prior to its conduction.

\section{$+\underline{\text { Teacher registration }}$}

Before creating an educational escape room profile in the platform, teachers need to create an account on it. Enabling teachers to use their institutional credentials to log in to the platform would be an interesting feature.

\section{+ Basic information}

The platform must allow teachers to specify the name of the educational escape room, the course to which it belongs, and the place where it will take place, in order to correctly differentiate one escape room from another. The basic information may also include additional data such as a small description of the activity so that students know what to expect. 


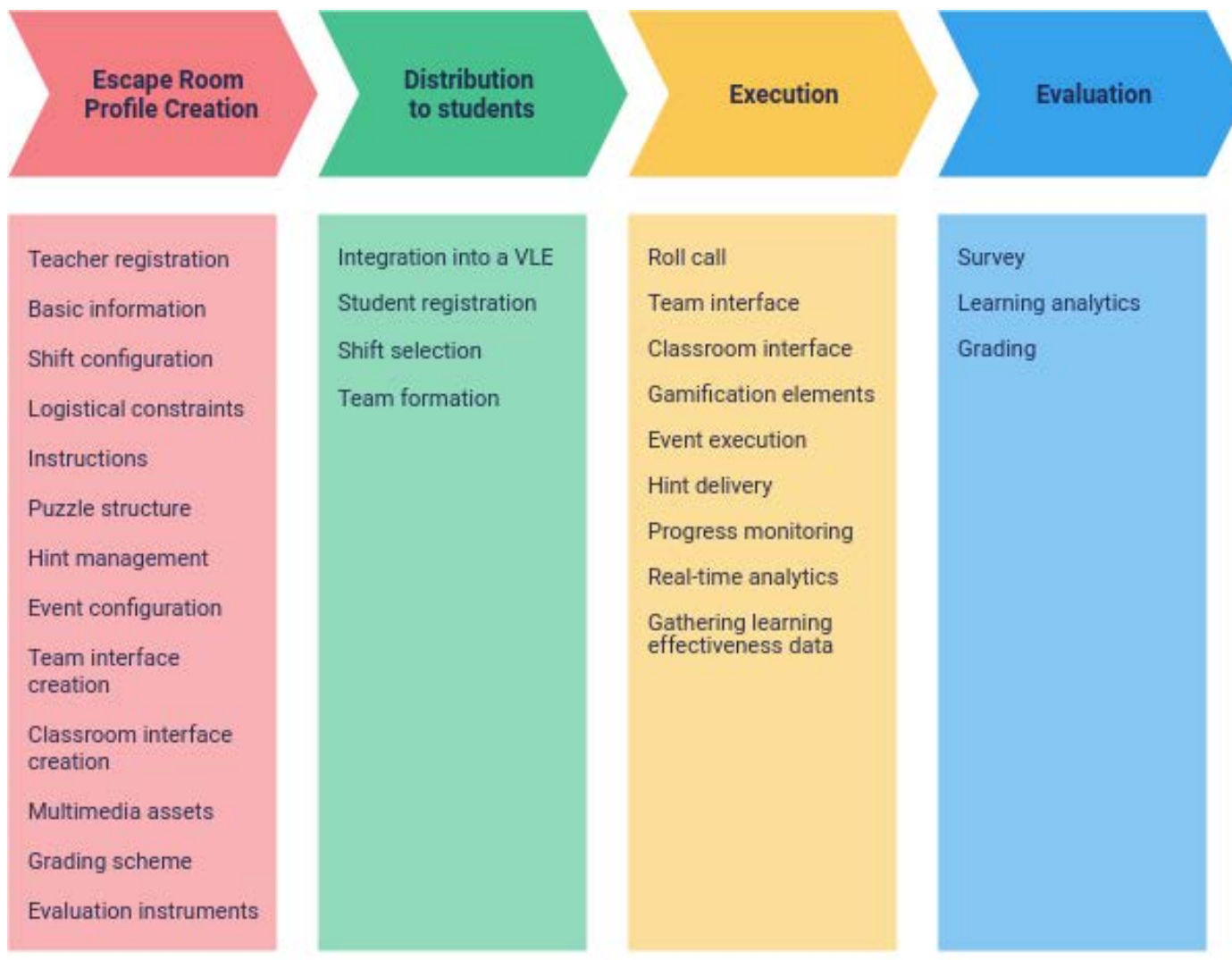

Figure 1. Requirement summary.

\section{+ Shift configuration}

Since conducting an educational escape room in a single session can be an overwhelming task, especially for large courses, teachers may want to run different shifts of the same escape room. The platform should allow teachers to schedule different days and times, so students can choose the one that suits them better.

\section{+ Logistical constraints}

The platform needs to know the maximum number of participants and teams that are allowed to perform the activity at the same time (i.e. in each shift), the allowed team size, and the duration of the activity. Thereby, the platform will be able to enforce these constraints on students when the time comes to sign up for the escape room. It would also be useful if teachers could specify whether an educational escape room requires students to bring specific equipment (laptops, smartphones, headphones, scissors...).

\section{+ Instructions}

Before conducting an educational escape room, students need to be provided with initial instructions that explain what the activity consists of, indicate how to sign up, specify its rules (e.g. if participants are allowed to talk or exit the room) and assessment, indicate if some equipment (e.g. a laptop) needs to be brought, and provide any other information that students may need before participating. The platform should allow teachers to provide custom instructions for each escape room.

\section{+ Puzzle structure}

Regarding structure, escape rooms are frequently composed of a series of puzzles that need to be solved either in a linear sequence, in no particular order, or a mix of both. Introducing the puzzle structure in the platform allows knowing which puzzles need to be solved in order to unlock each puzzle. This way, the application is capable of providing students with the appropriate hints in each moment as well as to track the progress of each team in an accurate way. Linear escape rooms allow for a better follow-up of students' progress since, by knowing the number of puzzles solved, teachers can easily know exactly how long students have left, whereas escape rooms in which students can attempt to solve multiple puzzles simultaneously are more intricate. For the student tracking of an 
educational escape room to be feasible, besides allowing teachers to define the structure of the puzzles, the platform should allow them to define the solutions of the puzzles.

\section{+ Hint management}

During an educational escape room, students often get stuck and need help solving puzzles. In order to deal with this issue, the platform should allow students to get hints for specific puzzles. It should be possible to deliver these hints when requested by students or provide them proactively under certain circumstances (for instance, when a team is lagging). Furthermore, these hints should be able to be delivered automatically or provided by the teachers supervising the activity. In order to provide hints automatically, it is necessary that, prior to the activity, teachers specify a series of hints for each puzzle through the platform. Different approaches can be used to deliver hints to students during an educational escape room. For instance, hints can be provided for free when students need assistance [9], [18]-[20], be handed out in exchange for something like a hint card [13], [15], delivered after students pass a short quiz [8], or be scheduled at specific moments of the escape room [10], [14]. Ideally, a platform for managing educational escape rooms should be able to support all the aforementioned approaches for delivering hints, as well as hybrid approaches. Besides, in order to properly support the hint approach in which students must pass a quiz in order to get a hint, the platform should allow teachers to insert the quiz questions through files compliant with common e-Learning specifications such as IMS QTI [26] and Moodle XML [27].

\section{+ Event configuration}

Another useful feature for the platform would be to allow teachers to program certain events to happen at specific instants during the escape room. For instance, it is common to play a video at the very beginning in order to introduce the students to the narrative and present the objective they need to accomplish. Another possibility is to play a fitting soundtrack in the background during the activity in order to create a certain atmosphere. Other events that teachers could configure are, for example, offering certain teams a free hint at a specific moment, reminding of the time that is left every 15 minutes, and showing an additional video with a plot twist at halftime or when a team completes a certain puzzle. Although an event will generally occur for all teams at the same time, on some occasions it is necessary to trigger events for specific teams. Thus, this requirement should be satisfied by the platform as well.

\section{$+\underline{\text { Team interface creation }}$}

Although the actual content and puzzles of the educational escape rooms are not displayed on the platform, it is necessary to provide teams with an interface they can access while conducting an educational escape room through which they can check the countdown and their progress, gain access to hints, introduce puzzle solutions, view additional multimedia assets and interact with the platform in any other way. Teachers should be capable of customizing this interface beforehand by arranging all the components in their preferred disposition and adjusting its appearance in order to fit the narrative of the escape room.

\section{+ Classroom interface creation}

In addition to an interface for teams, the platform should allow teachers to configure a screen that they can project in the classroom while an educational escape room takes place. Teachers should be able to decide which elements will appear on this screen and the appearance of it. The elements that the platform should allow adding to this screen include text, pictures, videos (for instance, to show the introductory video at the beginning), the countdown, and gamification elements such as a leaderboard or badges. It should be also possible to change the content of the screen in accordance with the state (not started, started and finished) of the educational escape room.

\section{+ Multimedia assets}

A useful feature for a platform aimed at managing educational escape rooms would be to provide teachers with a way of adding different assets (videos, audios, PDF files, ...) involved in the activity. These assets can be a part of the puzzles of the escape room (e.g. a picture file showing a map) or merely contribute to its ambience (e.g. an audio file used as background music). Different types of assets should be supported through files or URLs including videos, audios, pictures and text documents. 


\section{+ Grading scheme}

The platform should allow teachers to define the grading scheme of the educational escape rooms by selecting the different aspects to be taken into account for calculating students' grades and assigning weights to each one of them. Useful aspects to be considered in the grading of an educational escape room include attendance, puzzle completion (different puzzles may have different weights), number of hints requested, position on the leaderboard, and time spent to complete the activity. By taking into account all of these variables when grading students, teachers can provide them with a grade proportional to their effort and performance.

\section{+ Evaluation instruments}

In order to evaluate the learning effectiveness and student acceptance of an educational escape room, the platform should allow teachers to create and/or upload, for each escape room, a pre-test and post-test, and a student survey.

\subsection{Distribution to students}

The distribution phase encompasses the period from the moment that the teacher has finished creating the educational escape room profile in the platform until the day that the activity takes place. During this phase, students must sign up in order to participate in the escape room.

\section{+ Integration into a VLE}

The preferable way of allowing students to join an educational escape room is to integrate the activity into the VLE they use for accessing the other activities and materials of the course. This way, students are capable of accessing the educational escape room in the same way they access the other learning activities in the course. Another alternative that teachers have for distributing an educational escape room activity is by providing students with a link to it, for instance, through email, messaging systems or QR codes projected in the classroom.

In order to integrate an educational escape room into a VLE, it would be convenient to use some content integration e-Learning standard such as SCORM [28], XAPI [29] or LTI [30]. This way, students' data (e.g. progress and grade) could be automatically reported to the VLE, and the platform would be able to access students' information provided by the VLE. Taking into account that major content integration e-Learning standards have been developed to facilitate the integration of web resources into VLEs, it becomes clear that a platform for managing educational escape rooms should be web-based.

Besides allowing students to access an educational escape room, it is also necessary to provide them with the initial instructions the teacher elaborated for the activity. These instructions can be easily provided through a VLE as an additional resource, through the educational escape room management platform prior to the activity, or in other ways such as by email or printed.

\section{+ Student registration}

Students need to sign up for an educational escape room in advance in order to participate in it. Depending on how the activity has been integrated into the VLE, students could need to log in or register on the platform before signing up for the escape room. In this regard, it would be very useful if, instead of having to create an account from scratch, students could use their institutional credentials to access the escape room management platform. The platform could also allow teachers to sign all the students of a course up for an educational escape room in bulk by providing a list with all the students' identifiers.

\section{$+\underline{\text { Shift Selection }}$}

When students access the profile of an educational escape room in the platform, they should be presented with an interface to select the shift they prefer from the shifts that the teacher has previously set up.

\section{+ Team formation}

The platform should allow students to join an existing team or create their own team for their peers to join later. The platform should also allow students to sign up for an educational escape room without a team and automatically form teams for these students afterwards. It would also be useful to enable teachers to create the teams instead of students. 


\subsection{Execution}

The execution phase of an educational escape room encompasses all the actions that take place the day of the activity from the moment in which students start to when they finish.

\section{+ Roll call}

The day of the activity teachers should be able to use the platform to take student attendance so as to keep control of who participates in the escape room. This requirement is of special interest if attendance to the escape room is mandatory or if it has an impact on students' grades.

\section{$+\underline{\text { Team interface }}$}

Students should be able to access the aforementioned team interface through the platform during the conduction of an educational escape room. This interface should enable students to view the current progress of their teams, check the countdown, obtain and/or request hints, introduce puzzle solutions (if necessary), view additional multimedia assets provided by the teachers, and interact with the platform in any other way needed to allow students complete the escape room.

\section{$+\underline{\text { Classroom interface }}$}

While an educational escape room is running, the platform should allow teachers to project the classroom interface previously described. Thereby, different elements could be shown to all students participating in an educational escape room, including videos, messages, the countdown, and gamification elements (described in the next section).

\section{+ Gamification elements}

It is common in educational competitive activities to use gamification elements such as leaderboards and badges. A leaderboard showing the names and current progress (and/or points) of the teams helps trigger competition among students and contributes to the feeling of tension that the escape room aims to create. Badges can be used to reward teams, for instance, when they are the first ones or the fastest ones in solving a certain puzzle, or if they are the team that needed fewer hints to complete the escape room. Teachers should be able to display all the gamification elements that they have included in the escape room on the classroom interface if they want to show them to all the participants, or on the team interface, if they are only meant for a specific team.

\section{+ Event execution}

The platform should be able to automatically trigger all the events configured by the teachers in the profile of the educational escape room previously created in the platform. These events include video and audio playing, message viewing, background music playing, and delivery of scheduled hints. An event could update the team interface, the classroom interface or both of them. Additionally, the platform should provide a way for teachers to officially start the educational escape room, commencing the countdown and giving students access for the first time to the team interface.

\section{+ Hint delivery}

The platform should guarantee that, during an educational escape room, hints can be delivered to students according to the hint approach specified by the teacher in the profile of the escape room created in the platform. If the hint approach allows students to request hints, they should be able to do that from the team interface. In this case, if multiple puzzles are available for students to attempt at a certain stage of an educational escape room (i.e. if at a certain time there are several unlocked puzzles that have been not completed yet), students should be able to choose through the team interface for which one of them they would like to request the hint. If automatic hints are provided, they should become available at the team interface for students to consume and review. The team interface could also show additional information to students (which will depend on the hint approach used in the educational escape room) including, for example, the hint cards available and consumed (if any), the total number of hints requested, the number of quizzes attempted, etc. If a hint approach requires hints to be provided proactively under certain circumstances, the platform must be able to automatically detect such circumstances. For instance, if a hint should be automatically delivered to a team that is behind, the platform needs to monitor team progress and be able to decide, based on that progress, when a team is lagging or has been left behind. 


\section{+ Progress monitoring}

Automatically monitoring students' progress is crucial for teachers to know what is going on during an educational escape room and for its subsequent assessment. Besides, since delivering hints automatically requires the platform to know which puzzles have been solved by each team, this requirement should also be met in order to allow such delivery. Moreover, student progress monitoring is also necessary for deciding when some events should be triggered (e.g. when a free hint should be given to a lagging team) as well as for some gamification elements.

In an educational escape room, the progress of the students depends mainly on the puzzles that have been solved by their corresponding teams. Each of the puzzles can contribute to the total progress measure of the educational escape room with a different weight. There are two main ways in which the platform can become aware of the puzzles that each team has successfully completed: (1) through the requests sent from the team interface, and (2) through the requests sent to a puzzle checker API. On the one hand, when students introduce the correct solution to a puzzle through the team interface and check that solution, this information is automatically recorded by the platform. On the other hand, if a puzzle of an educational escape room relies on a software application, this application can automatically send a remote request to the platform when the puzzle has been completed. For this to be possible, the platform needs to expose an API that allows applications to report that a certain puzzle has been completed by a certain team. It should be pointed out that, in order to use this API, applications need to be able to identify the team that is using them. A possible approach to deal with this issue would be to specify in advance the team identifiers through environment variables in the devices that students will use during the activity.

\section{+ Real-time analytics}

Real-time analytics can be very helpful for teachers to have a view of what is going on during gamified educational activities, in order to be able to react to given situations, as pointed out by [31]. In an educational escape room, providing these analytics to teachers in an effective way (e.g. through dashboards), they will be able to make decisions such as offering a free hint to all the teams or only to lagging teams, or to extend the time initially scheduled for the activity. It would also be useful for the platform to proactively suggest interventions to teachers based on real-time analytics and students' information. In order to generate real-time analytics, beyond students' progress data, the platform should record additional data such as the time each team spent on each puzzle and the number of hints requested and/or provided to each team.

\section{+ Gathering learning effectiveness data}

One of the biggest questions regarding educational escape rooms is whether they actually help students improve their current skills and knowledge of the course materials. In order to help answer this question, the platform should be able to require students to complete a pre-test (right before the activity) and a post-test (right after the activity) so teachers can later analyse if there has been a learning impact. If completing the pre-test is mandatory, the platform should not allow students to move forward within the escape room if they have not completed it.

\subsection{Evaluation}

The evaluation phase of the escape room is the last phase and takes place once the activity has finished.

\section{+ Survey}

Student opinion assessment can be easily done by conducting a survey asking for students' thoughts on the key aspects of the escape room. The platform should be able to present a survey to students once they finish the activity.

\section{+ Learning analytics}

A lot of useful information can be extracted from students' interaction data after an educational escape room is finished. For instance, teachers can find out at which puzzles students get stuck more often, so they can dedicate more time in class to review the concepts covered in those puzzles. The platform should present all the information gathered at run-time in a way that is easy to understand for teachers (e.g. by using dashboards) so they are capable of analysing the results after the activity and act upon them. Moreover, teachers should be able to download this information as spreadsheet files. 


\section{+ Grading}

The platform should allow teachers to calculate students' grades based on the grading scheme set up beforehand. If an educational escape room has been integrated into a VLE using an e-Learning content integration standard that allows to report grades, students' grades could be reported automatically. Otherwise, if grades are not reported automatically, the platform should allow teachers to export the grades to a format compliant with the course VLE. Easy grade transferring from gaming software to VLEs has been a key aspect of prior research in facilitating the integration of games in the classroom [32], [33]. Another interesting feature that the platform could have if automatic grade reporting is not supported is the possibility to preview and recalculate grades, allowing teachers to tweak the weights assigned to the different variables that make up the final grade if necessary.

\section{CONCLUSIONS}

This work identifies and analyses for the first time the requirements that a platform for managing educational escape rooms should meet in order to allow teachers to cover all the aspects of managing activities of this kind. The requirements identified were divided into four groups, each of them related to one of the phases involved in the management of an educational escape room through the platform: creation of a profile for the educational escape room including all the necessary information, distribution to students, execution of the activity, and evaluation. Taking into account that managing educational escape rooms is a complex and laborious task for teachers that could be eased by using appropriate tools, and that no previous studies have reported on the use, development or design of any tool for managing educational escape rooms, this paper makes an important contribution by identifying and analysing a set of requirements that could be used in the future for the development of an effective platform for managing educational escape rooms. This platform would help to overcome one of the main barriers hampering the use and adoption of educational escape rooms.

Based on the requirements identified in this work, we plan to develop a web platform to help teachers manage educational escape rooms. The platform developed will be evaluated through its use in educational escape rooms conducted in higher education settings both by students and teachers. Taking a leap forward, it would be extremely useful to develop tools to provide assistance to teachers, not only when managing educational escape rooms but also at the time of designing and building them. In this regard, an interesting future work would be to develop resources and tools to ease the building of both physical and digital escape room puzzles. If the digital puzzles created through these tools use the puzzle checker API proposed in this work, it would be possible to automatically track students' progress.

\section{ACKNOWLEDGEMENTS}

This work has been supported by Universidad Politécnica de Madrid (Spain) through the Educational Innovation Project with reference IE1819.0905.

\section{REFERENCES}

[1] S. Nicholson, "Peeking behind the locked door: A survey of escape room facilities," 2015. [Online]. Available: http://scottnicholson.com/pubs/erfacwhite.pdf. [Accessed: 09-Sep-2019].

[2] R. Pan, H. Lo, and C. Neustaedter, "Collaboration, Awareness, and Communication in Real-Life Escape Rooms," in Proceedings of the 2017 Conference on Designing Interactive Systems (DIS '17), 2017, pp. 1353-1364.

[3] H. Warmelink et al., "AMELIO: Evaluating the Team-building Potential of a Mixed Reality Escape Room Game," in Extended Abstracts Publication of the Annual Symposium on Computer-Human Interaction in Play (CHI PLAY' 17), 2017, pp. 111-123.

[4] M. Wiemker, E. Elumir, and A. Clare, "Escape Room Games. Can you transform an unpleasant situation into a pleasant one?," 2015. [Online]. Available: https://thecodex.ca/wp-content/ uploads/2016/08/00511Wiemker-et-al-Paper-EscapeRoom-Games.pdf. [Accessed: 09-Sep-2019].

[5] C. Wu, H. Wagenschutz, and J. Hein, "Promoting leadership and teamwork development through Escape Rooms," Medical Education, vol. 52, no. 5, pp. 561-562, 2018. 
[6] P. Williams, "Using escape room-like puzzles to teach undergraduate students effective and efficient group process skills," in Proceedings of the 2018 IEEE Integrated STEM Education Conference (ISEC 2018), 2018, pp. 254-257.

[7] C. McFadden and S. Porter, "Augmented reality escape rooms as high-engagement educational resources," in Proceedings of the 11th International Conference of Education, Research and Innovation (ICERI 2018), 2018, pp. 4361-4365.

[8] S. Lopez-Pernas, A. Gordillo, E. Barra, and J. Quemada, "Examining the Use of an Educational Escape Room for Teaching Programming in a Higher Education Setting," IEEE Access, vol. 7, pp. 31723-31737, 2019.

[9] V. Adams, S. Burger, K. Crawford, and R. Setter, "Can You Escape? Creating an Escape Room to Facilitate Active Learning," Journal for Nurses in Professional Development, vol. 34, no. 2, pp. E1-E5, 2018.

[10] J. L. Gómez-Urquiza, J. Gómez-Salgado, L. Albendín-García, M. Correa-Rodríguez, E. GonzálezJiménez, and G. A. Cañadas-De la Fuente, "The impact on nursing students' opinions and motivation of using a 'Nursing Escape Room' as a teaching game: A descriptive study," Nurse Education Today, vol. 72, pp. 73-76, 2019.

[11] S. R. Monaghan and S. Nicholson, "Bringing Escape Room Concepts to Pathophysiology Case Studies," HAPS Educator, vol. 21, no. 2, pp. 49-65, 2017.

[12] M. Boysen-Osborn, S. Paradise, and J. Suchard, "The Toxiscape Hunt: An Escape RoomScavenger Hunt for Toxicology Education," Journal of Education and Teaching in Emergency Medicine, vol. 3, no. 1, pp. 9-19, 2018.

[13] M. Hermanns et al., 'Using an 'Escape Room' toolbox approach to enhance pharmacology education," Journal of Nursing Education and Practice, vol. 8, no. 4, pp. 89-95, 2018.

[14] J. Cain, "Exploratory implementation of a blended format escape room in a large enrollment pharmacy management class," Currents in Pharmacy Teaching and Learning, vol. 11, no. 1, pp. 44-50, 2019.

[15] H. N. Eukel, J. E. Frenzel, and D. Cernusca, "Educational Gaming for Pharmacy Students Design and Evaluation of a Diabetes-themed Escape Room.," American Journal of Pharmaceutical Education, vol. 81, no. 7, 2017.

[16] S. Cotner, K. M. Smith, L. Simpson, D. S. Burgess, and J. Cain, "1311. Incorporating an 'Escape Room' Game Design in Infectious Diseases Instruction," Open Forum Infectious Diseases, vol. 5, pp. S401-S401, 2018.

[17] C. Jiménez, R. Lafuente, M. Ortiz, L. Bruton, and V. Millán, "Room Escape: Propuesta de Gamificación en el Grado de Fisioterapia," in Actas del III Congreso Nacional de Innovación Educativa y de Docencia en Red (IN-RED 2017), 2017, pp. 537-551.

[18] N. Dietrich, "Escape Classroom: The Leblanc Process-An Educational 'Escape Game," Journal of Chemical Education, vol. 95, no. 6, pp. 996-999, 2018.

[19] C. Borrego, C. Fernández, I. Blanes, and S. Robles, "Room escape at class: Escape games activities to facilitate the motivation and learning in computer science," Journal of Technology and Science Education, vol. 7, no. 2, pp. 162-171, 2017.

[20] A. M. Ho, "Unlocking Ideas: Using Escape Room Puzzles in a Cryptography Classroom," PRIMUS, vol. 28, no. 9, pp. 835-847, 2018.

[21] J. Melero and D. Hernández-Leo, "A Model for the Design of Puzzle-based Games Including Virtual and Physical Objects," Educational Technology \& Society, vol. 17, no. 3, pp. 192-207, 2014.

[22] G. Guigon, M. Vermeulen, and J. Humeau, "A Creation Tool for Serious Puzzle Games," in Proceedings of the 11th International Conference on Computer Supported Education (CSEDU 2019), 2019, pp. 556-561.

[23] D. Pranantha, F. Bellotti, R. Berta, and A. De Gloria, "Puzzle-it: An HTML5 Serious Games Platform for Education," in E-Learning and Games for Training, Education, Health and Sports. Edutainment 2012, GameDays 2012. Lecture Notes in Computer Science, vol. 7516., S. Göbel, W. Müller, B. Urban, and J. Wiemeyer, Eds. Springer Berlin Heidelberg, 2012, pp. 134-143. 
[24] P. Moreno-Ger, I. Martinez-Ortiz, V. Francisco Gilmartin, and R. Hervás Ballesteros, "TrivialCV: Competitive Activities for the Classroom Integrated in a Moodle Virtual Campus," IEEE Revista Iberoamericana de Tecnologias del Aprendizaje, vol. 8, no. 1, pp. 31-38, Feb. 2013.

[25] Y. Chaiyo and R. Nokham, "The effect of Kahoot, Quizizz and Google Forms on the student's perception in the classrooms response system," in Proceedings of the 2017 International Conference on Digital Arts, Media and Technology (ICDAMT 2017), 2017, pp. 178-182.

[26] "IMS Question \& Test Interoperability (QTI) Specification," IMS Global Learning Consortium. [Online]. Available: http://www.imsglobal.org/question/index.html. [Accessed: 09-Sep-2019].

[27] "Moodle XML format," Moodle. [Online]. Available:

https://docs.moodle.org/33/en/Moodle_XML_format. [Accessed: 09-Sep-2019].

[28] "SCORM 2004 4th Edition," ADL (Advanced Distributed Learning) Initiative. [Online]. Available: http://www.adlnet.gov/adl-research/scorm/scorm-2004-4th-edition. [Accessed: 09-Sep-2019].

[29] "Experience API (XAPI)." [Online]. Available: http://experienceapi.com. [Accessed: 09-Sep-2019].

[30] "IMS Learning Tools Interoperability Specification," IMS Global Learning Consortium. [Online]. Available: http://www.imsglobal.org/activity/learning-tools-interoperability. [Accessed: 09-Sep2019].

[31] J. Marty and T. Carron, "Observation of Collaborative Activities in a Game-Based Learning Platform," IEEE Transactions on Learning Technologies, vol. 4, no. 1, pp. 98-110, 2011.

[32] J. W. Kemp, D. Livingstone, and P. R. Bloomfield, "SLOODLE: Connecting VLE tools with emergent teaching practice in Second Life," British Journal of Educational Technology, vol. 40, no. 3, pp. 551-555, 2009.

[33] P. Castro, G. Matas, I. Luque, and M. A. Gomez-Nieto, "Use of NFC-based Pervasive Games for Encouraging Learning and Student Motivation," in Proceedings of the 3rd International Workshop on Near Field Communication, 2011, pp. 32-37. 